\title{
Duhuo Jisheng decoction inhibits endoplasmic reticulum stress in chondrocytes induced by tunicamycin through the downregulation of miR-34a
}

\author{
FAYUAN LIU ${ }^{1,4}$, XIAPING WENG ${ }^{2}$, PINGDONG LIN ${ }^{2}$, CHUNSONG ZHENG $^{1,3}$, \\ HUIFENG XU ${ }^{1,3}$, XIANXIANG LIU ${ }^{1}$, HONGZHI YE ${ }^{1}$ and XIHAI LI ${ }^{1}$ \\ ${ }^{1}$ Academy of Integrative Medicine, ${ }^{2}$ College of Pharmacy, ${ }^{3}$ Fujian Key Laboratory \\ of Integrative Medicine on Geriatrics, Fujian University of Traditional Chinese Medicine, \\ Fuzhou, Fujian 350122; ${ }^{4}$ Putian Xiuyuqu Hospital, Putian, Fujian 351146, P.R. China
}

Received February 11, 2015; Accepted July 13, 2015

DOI: $10.3892 / \mathrm{ijmm} .2015 .2331$

\begin{abstract}
Our previous study showed that Duhuo Jisheng decoction (DHJSD) inhibited chondrocyte apoptosis by the mitochondria-dependent signaling pathway. Endoplasmic reticulum (ER) stress is upstream of the mitochondria-dependent signaling pathway and has been shown to promote chondrocyte apoptosis that occurs in osteoarthritis (OA). The present study aimed to evaluate whether DHJSD inhibits the chondrocyte apoptosis by regulating ER stress. DHJSD enhanced the viability of tunicamycin (TM)-exposed chondrocytes, a model of ER stress-induced apoptosis, in a dose- and time-dependent manner, as shown by MTT assay. The present results showed that DHJSD and sodium 4-phenylbutyrate (PBA), an ER stress inhibitor, reduced TM-induced chondrocyte apoptosis by 4',6-diamidino-2-phenylindole staining. To gain insight into the mechanisms of DHJSD that are responsible for enhancing the viability and inhibiting TM-induced chondrocyte apoptosis, the associated mRNA expressions and protein levels were detected by reverse transcription-polymerase chain reaction (RT-PCR) and western blot analysis, respectively. The results showed that the expression levels of Xbp1, Xbp1s and Bcl-2 were increased, and the expression levels of Bip, Atf4, Chop, Bax, caspase- 9 and -3 were decreased in the TM-exposed chondrocytes treated with DHJSD or PBA compared with that in the TM-exposed chondrocytes. To identify the possible mechanisms, the expression of miR-34a was examined by the TaqMan microRNA assay, and was downregulated in the TM-exposed chondrocytes treated with DHJSD or PBA
\end{abstract}

Correspondence to: Professor Hongzhi Ye or Dr Xihai Li, Academy of Integrative Medicine, Fujian University of Traditional Chinese Medicine, 1 Qiuyang, University Town, Fuzhou, Fujian 350122, P.R. China

E-mail: yelin0930@163.com

E-mail: lixihai79dahai@163.com

Key words: Duhuo Jisheng decoction, chondrocyte, endoplasmic reticulum stress, apoptosis, miR-34a compared with that in the TM-exposed chondrocytes. DHJSD inhibits ER stress in chondrocytes induced by exposure to TM by downregulating miR-34a, suggesting that DHJSD may be a potential therapeutic agent for OA.

\section{Introduction}

Osteoarthritis (OA) is a progressive cartilage degradation skeletal disease characterized by the synthesis and degradation imbalance of extracellular matrix (ECM), resulting in a gradual loss of cartilage integrity (1). The final common pathway of cartilage degradation arises from the failure of chondrocytes to maintain the homeostatic balance between ECM synthesis and degradation (2). Chondrocytes are the only cell type resident in the cartilage and it has been demonstrated that the increasing number of chondrocytes undergoing apoptosis is significantly correlated with the severity of OA $(3,4)$. Recently, endoplasmic reticulum (ER) stress was found to have a crucial role in apoptosis (5), and is considered to be one of the most important risk factors for the pathology during OA progression (6).

ER stress can initiate apoptosis by diverse stimuli. Upon ER stress, three sensors, protein kinase RNA-like ER kinase (Perk), activating transcription factor-6 (Atf6) and inositol requiring protein-1 (Ire1), are primarily activated, leading to the initiation of unfolded protein response (UPR). Persistent ER stress or UPR occurs from failure to correct the balance, which activates the cell apoptotic program $(7,8)$, leading to caspase-mediated apoptosis (9). ER stress has been shown to increase chondrocyte apoptosis and to decrease the proteins of ECM, including type II collagen and aggrecan, which cause a vicious cycle of cartilage degradation $(10,11)$. Additionally, it has been demonstrated that microRNAs (miRNAs or miRs) are involved in survival and apoptosis $(12,13)$, and miR-34a plays a pivotal role in chondrocyte apoptosis (14). The effect of delaying chondrocyte apoptosis by regulating ER stress may be one of the therapeutic strategies to treat OA.

Duhuo Jisheng decoction (DHJSD), a traditional Chinese herbal formula, confers the effects of expelling wind-dampness, relieving numbness and pain, nourishing the liver and kidneys, invigorating $q i$-blood, and has been used for treating 
OA and proved effective by relieving pain, reducing joint stiffness, and improving mobility and quality of life (15). Previous studies have proven that DHJSD has potential cooperation and polypharmacology against OA, and could inhibit chondrocyte apoptosis by the mitochondria-dependent signaling pathway (16). However, the mechanisms of how DHJSD inhibit the chondrocyte apoptosis by ER stress remain to be elucidated, which has limited its wider use. In the present study, the effects and cellular mechanisms of DHJSD were investigated on ER stress tunicamycin (TM)-induced chondrocyte apoptosis. Furthermore, the potential mechanisms of action of DHJSD on ER stress apoptosis were examined by measuring the expression of miR-34a.

\section{Materials and methods}

Preparation of DHJSD aqueous extract. DHJSD is composed of $9 \mathrm{~g}$ of Radix Angelicae Pubescentis and $6 \mathrm{~g}$ of Radix Gentianae Macrophyllae, Ramulus Loranthi, Radix Saposhnikoviae, Herba Asari, Cortex Cinnamomi, Poria Cocos, Rhizoma Chuanxiong, Radix Angelicae Sinensis, Radix Achyranthis Bidentatae, Radix Rehmanniae Preparata, Radix Paeoniae Alba, Cortex Eucommiae Ulmoidis, Panax ginseng and Radix Glycyrrhizae. The herbs were identified by the teaching and research section of Fujian University of Traditional Chinese Medicine (TCM; Fuzhou, China) and the components were mixed and extracted with the standard methods according to Chinese Pharmacopoeia (China Pharmacopoeia and Committee, 2010). Herbs were soaked in distilled water and boiled for 30 min twice, and the extracts were filtered and concentrated. The concentrate filtrate was dissolved in Dulbecco's modified Eagle's medium (HyClone, Logan, UT, USA) at a concentration of $10 \mathrm{mg} / \mathrm{ml}$, and was subsequently filtered and stored at $4^{\circ} \mathrm{C}$.

The quality control of the DHJSD extracts was analyzed by the contents of polysaccharides and coumarins using an ultraviolet (UV) spectrophotometer (Beckman Coulter, Inc., Brea, CA, USA). Concentration gradients of the glucose standard solutions (National Institutes for Food and Drug Control, China) and DHJSD were prepared and subsequently measured at $490 \mathrm{~nm}$ using phenol-sulphate colorimetry. A series concentration of osthole standard solutions (National Institutes for Food and Drug Control, China) and the DHJSD were dissolved in methanol, respectively, and detected at $320 \mathrm{~nm}$.

Isolation, identification and treatment of chondrocytes. The present study was approved by the Institutional Animal Care and Use Committee of Fujian University of TCM. Male Sprague-Dawley rats (4-week-old) were from Super-BK Laboratory Animal Co. (Shanghai, China). Chondrocytes were isolated from articular cartilage and cultured as previously described (17). The second-passage (P2) chondrocytes cultured until $\sim 80 \%$ confluency were used in the study.

Assessment of cell viability. Chondrocytes were seeded at a density of $5 \times 10^{4}$ cells $/ \mathrm{ml}$ in 96 -well plates $(100 \mu \mathrm{l} /$ well $)$ for $24 \mathrm{~h}$. Subsequently, the medium was replaced with or without $2 \mu \mathrm{g} / \mathrm{ml}$ TM (Sigma-Aldrich, St. Louis, MO, USA) and a series of concentrations of DHJSD (50, 100, 200, 300 and $400 \mu \mathrm{g} / \mathrm{ml}$ ) in the presence of $\mathrm{TM}$, and incubated for 24 ,
48 or 72 h. Following treatment, $100 \mu 1$ 1\% 3-(4,5-dimethylthiazol-2-yl)-2,5-diphenyltetrazolium bromide (MTT; Sigma-Aldrich) replaced the medium. After incubation at $37^{\circ} \mathrm{C}$ for $4 \mathrm{~h}$, the supernatant was replaced with $150 \mu \mathrm{l}$ dimethyl sulfoxide and shaked for $10 \mathrm{~min}$. The optical density (OD) was analyzed by measuring at $490 \mathrm{~nm}$ using an enzyme-linked immunosorbent assay reader (BioTek, Winooski, VT, USA).

Experimental design. The cells were assigned to 4 groups as follows: untreated cells, TM-exposed chondrocytes, TM-exposed chondrocytes treated with $200 \mu \mathrm{g} / \mathrm{ml}$ DHJSD, and TM-exposed chondrocytes treated with $5 \mathrm{mM}$ sodium 4-phenylbutyrate (PBA; Sigma-Aldrich), which was diluted in PBS.

Assessment of chondrocyte apoptosis by 4,6-diamidino-2-phenylindole (DAPI) staining. Following treatment, cells were washed with phosphate-buffered saline (PBS), and fixed with $4 \%$ neutral formaldehyde at $4^{\circ} \mathrm{C}$ for $15 \mathrm{~min}$. Subsequently, the cells were stained in $5 \mu \mathrm{g} / \mathrm{ml}$ DAPI for 5 min and washed 3 times with PBS, and were observed under a fluorescent microscope (FACSCalibur; Becton-Dickinson, San Jose, CA, USA).

Reverse transcription-polymerase chain reaction ( $R T-P C R)$. Total RNA was extracted from the cells using TRIzol reagent (Invitrogen, Grand Island, NY, USA). RNA (1 $\mu \mathrm{g})$ was reverse transcribed into cDNA according to the manufacturer's instructions. The DNA bands were analyzed by gel electrophoresis (1.5\% agarose) and examined using a Gel Documentation System (Bio-Rad, Hercules, CA, USA), subsequently normalized to that of $\beta$-actin. Primer sequences were as follows: Binding protein (Bip) forward, 5'-ATC AAC CCA GAT GAG GCT GTA GCA-3' and reverse, 5'-AGA CCT TGA TTG TTA CGG TGG GCT-3'; Atf4 forward, 5'-AAT GGCTGG CTA TGG ATG GG-3' and reverse, 5'-TGT CTG AGG GGG CTC CTT ATT AG-3'; X-box binding protein-1 (Xbp1) forward, 5'-AGC ATA GGC CTG TCT GCT TCA CTA-3' and reverse, 5'-TGG TAA AGT CCA GCA CTT GGG AGT-3'; Xbp1s forward, 5'-TCT GCT GAG TCC GCA GCA GG-3' and reverse, 5'-CTC TAA GAC TAG AGG CTT GG-3'; Bax forward, 5'-GGC GAT GAA CTG GAC AAC-3' and reverse, 5'-TCC CGA AGT AGG AAA GGA G-3'; Bcl-2 forward, 5'-TGG CAT CTT CTC CTT CCC-3' and reverse, 5'-GGT ACA TCT CCC TGT TGA CG-3'; caspase-9 forward, 5'-GCC TCA TCATCA ACA ACG-3' and reverse, 5'-CTG GTA TGG GAC AGC ATC T-3'; caspase-3 forward, 5'-GGA CCT GTG GAC CTG AAA-3' and reverse, 5'-GGG TGC GGT AGA GTA AGC-3'; and $\beta$-actin forward, 5'-GAG AGG GAA ATC GTG CGT GAC-3' and reverse, 5'-CAT CTG CTG GAA GGT GGA CA-3'.

Western blot analysis. Total protein was extracted from cells using radioimmunoprecipitation assay buffer, and protein concentrations were measured using a bicinchoninic acid kit. An equal amount of protein was separated by electrophoresis on sodium dodecyl sulfate-polyacrylamide gel electrophoresis (SDS-PAGE) and was transferred onto PVDF membranes. Following blocking with 5\% non-fat milk, membranes were incubated with primary antibodies against Bip, Atf4, C/EBP-homologous protein (Chop), Xbp1, Bax, Bcl-2, caspase-3 and -9 (Cell Signaling Technology, Inc., Beverly, MA, USA) or $\beta$-actin (Santa Cruz Biotechnology, Inc., Dallas, TX, USA) at 

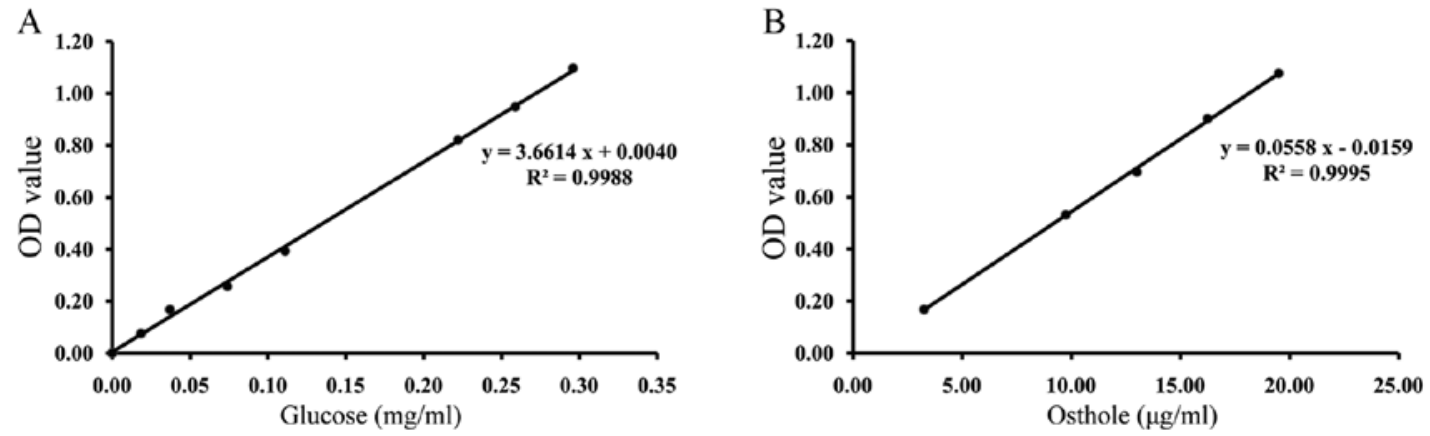

Figure 1. Quality control of the Duhuo Jisheng decoction extracts analyzed using ultraviolet radiation. (A) Concentration gradients of glucose standard solutions were measured at $490 \mathrm{~nm}$. (B) Series concentrations of osthole standard solutions were detected at $320 \mathrm{~nm}$.
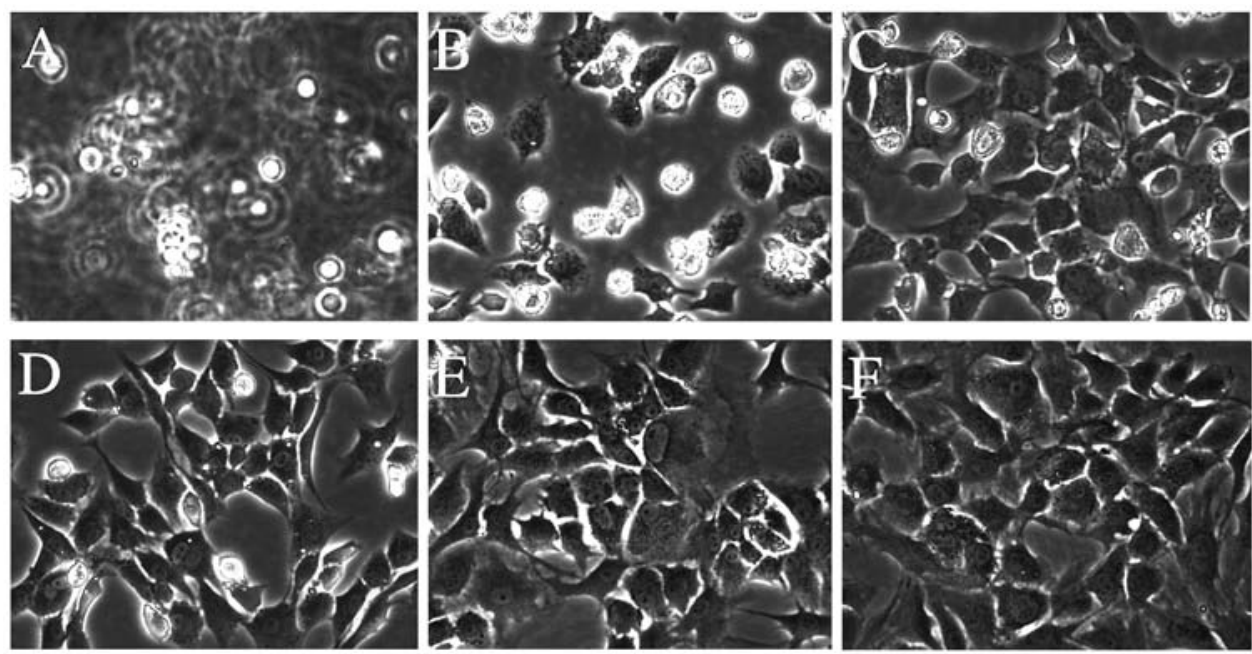

Figure 2. Morphology of the chondrocytes (magnification, x200). (A) Newly isolated chondrocytes. (B) Primary cells cultured for 3 days. (C) Primary cells cultured for 5 days. (D) First-passage cells cultured for 1 day. (E) First-passage cells cultured for 3 days. (F) Second-passage cells cultured for 3 days.

$4^{\circ} \mathrm{C}$ overnight followed by a horseradish peroxidase-conjugated secondary antibody (Zhongshan Goldenbridge Biotech, Beijing, China). ECL was used to make the blots visible, and blots were quantitated using a Bio-Rad Chemi Doc XRS ${ }^{+}$(Bio-Rad), normalizing to that of $\beta$-actin.

RT-quantitative (q) PCR. RT-qPCR was used to detect the expression of miR-34a. Total RNA was isolated according to the manufacturer's instructions for the mirVana ${ }^{\mathrm{TM}}$ isolation kit (Invitrogen, Life Technologies). Reverse transcription was performed with the TaqMan microRNA reverse transcription kit and miRNA specific stem-loop RT primers (both from Applied Biosystems, Foster City, CA, USA). The expression of miR-34a was confirmed by the TaqMan Universal PCR Master mix according to the manufacturer's instructions using a 7500 Real-time PCR System (both from Applied Biosystems). Fold changes were calculated by the formula $2^{-\Delta \Delta \mathrm{Ct}}$ relative to the expression in untreated cells and U6 was the endogenous control (18).

Statistical analysis. Data was analyzed by one-way analysis of variance or Student's t-test using SPSS 19.0 software (IBM Corp., Armonk, NY, USA) and expressed as mean \pm standard deviation from at least three independent experiments. $\mathrm{P}<0.05$ was considered to indicate a statistically significant difference.

\section{Results}

Quality control of DHJSD. UV is used for content determination of one type of material, which contains some of the same functional groups and has the same absorption wavelength in the UV spectrum. According to the regression equation of the glucose standard solutions (Fig. 1A) and osthole standard solutions (Fig. 1B), the contents of polysaccharides and coumarins were $1.69 \%$ and $0.0204 \%$, respectively. The extract quality of DHJSD could be controlled in this way.

Morphology and identification of chondrocytes. The morphology of newly isolated chondrocytes was small, round and floating in the medium, and after a few days culture, chondrocytes were connected during growth and showed an irregular paving stone shape with clear boundaries and distinct nuclei $(17,19,20)$ (Fig. 2). Type II collagen has been identified as the characterized form of collagen in articular cartilage, whereas proteoglycans provide the function of articular cartilage. To characterize the chondrocyte, the P2 chondrocytes cultured for 3 days were examined by type II collagen immunohistochemical staining and toluidine blue staining. The results showed that the brown-stained cytoplasm represented a positive expression of type II collagen, while the negative control did not stain brown by immunohistochemical staining, and 

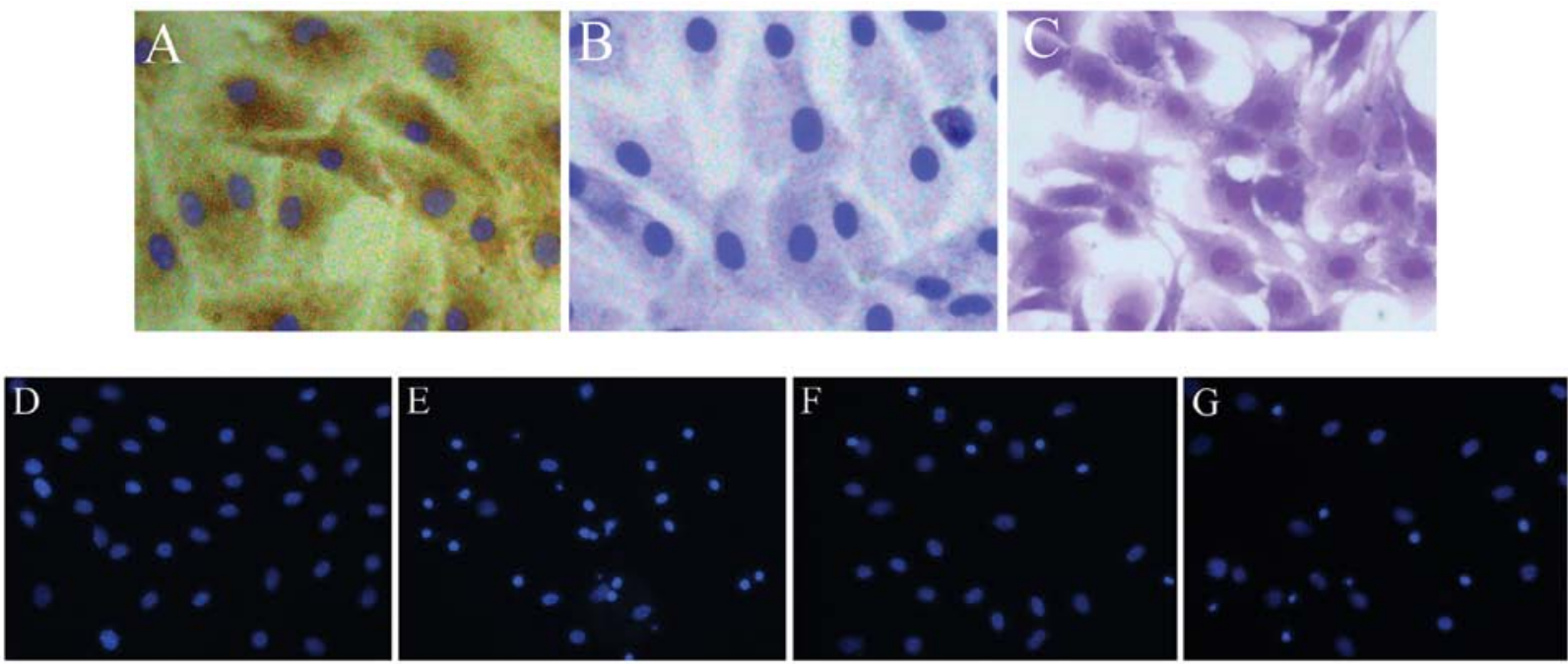

$\operatorname{TM}(2 \mu \mathrm{g} / \mathrm{ml})-$

$\operatorname{DHJSD}(\mu \mathrm{g} / \mathrm{ml}) 0$

PBA $(5 \mathrm{mM})$ -

$+$

0
200
$+$

0

Figure 3. Duhuo Jisheng decoction (DHJSD) reduces the apoptosis of tunicamycin (TM)-exposed chondrocytes. Second-passage chondrocytes cultured for 2 days, identified by (A) collagen type II immunohistochemistry, (B) negative control and (C) toluidine blue staining. Chondrocyte apoptosis morphology change was examined by 4',6-diamidino-2-phenylindole staining. (D) Untreated cells. (E) TM-exposed chondrocytes. (F) TM-exposed chondrocytes treated with $200 \mu \mathrm{g} / \mathrm{ml}$ DHJSD for $24 \mathrm{~h}$. (G) TM-exposed chondrocytes treated with $5 \mathrm{mM} 4$-phenylbutyrate (PBA) for $24 \mathrm{~h}$, as the positive control.
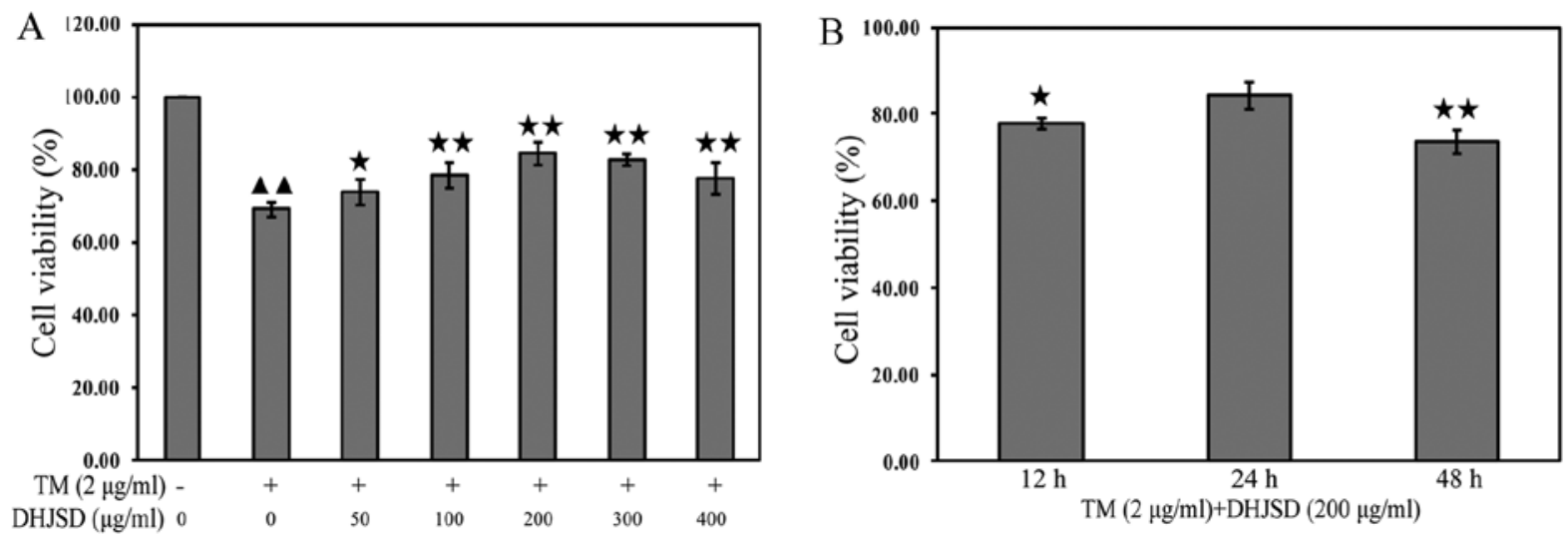

Figure 4. Duhuo Jisheng decoction (DHJSD) enhances the viability of tunicamycin (TM)-exposed chondrocytes. (A) Chondrocytes treated with or without TM and various concentrations of DHJSD in the presence of TM for $24 \mathrm{~h}$. Values are means \pm standard deviation (SD) and SD is shown as vertical bars. ${ }^{\wedge} \mathrm{P}<0.01$ compared to untreated cells (without TM and DHJSD); ${ }^{* *} \mathrm{P}<0.01$ and ${ }^{*} \mathrm{P}<0.05$ compared to TM-exposed chondrocytes. (B) TM-exposed chondrocytes treated with $200 \mu \mathrm{g} / \mathrm{ml}$ DHJSD for various periods of time. Values are means $\pm \mathrm{SD}$ and $\mathrm{SD}$ is shown as vertical bars. ${ }^{* *} \mathrm{P}<0.01$ and ${ }^{*} \mathrm{P}<0.05$ compared to $24 \mathrm{~h}$.

$\mathrm{red} /$ purple particles in the cytoplasm represent proteoglycans by toluidine blue staining (21) (Fig. 3A-C). P2 chondrocytes are rich in ECM and show a typical morphology of chondrocytes, and therefore, $\mathrm{P} 2$ chondrocytes were used in the subsequent experiments.

DHJSD enhances the viability chondrocytes exposed to TM. The effects of DHJSD on TM-exposed chondrocytes were evaluated by MTT assay. TM-exposed chondrocytes were treated with various concentrations of DHJSD for different times. The results showed that the viability of the TM-exposed chondrocytes was significantly lower than that of the untreated cells $(\mathrm{P}<0.01)$, and the viability of TM-exposed chondrocytes treated with DHJSD was higher than that of the TM-exposed chondrocytes $(\mathrm{P}<0.01$ and $\mathrm{P}<0.05)$ (Fig. $4 \mathrm{~A})$. The viability of TM-exposed chondrocytes treated with $200 \mu \mathrm{g} / \mathrm{ml}$ DHJSD for $24 \mathrm{~h}$ was higher than that of the 12- and 48-h treatment $(\mathrm{P}<0.01$ and $\mathrm{P}<0.05)$ (Fig. 3B), suggesting that DHJSD enhanced TM-exposed chondrocyte viability in a dose- and time-dependent manner. Therefore, $200 \mu \mathrm{g} / \mathrm{ml}$ DHJSD for $24 \mathrm{~h}$ was used in the following experiments.

DHJSD inhibits morphological changes in chondrocytes exposed to TM. The morphological changes of TM-exposed chondrocytes treated with or without DHJSD or PBA (Sigma-Aldrich) were observed by phase-contrast microscope (Fig. 5). The morphology of the untreated cells exhibited a healthy status, while TM-exposed chondrocytes presented more apoptotic cells that detached from each other and became bright, elongated and shrunken, or floated in the medium compared to that of the TM-exposed chondrocytes treated with DHJSD or PBA. 


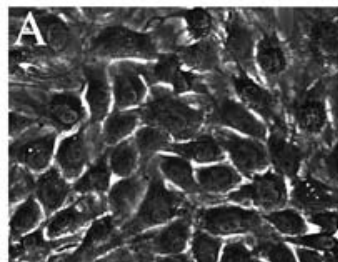

$\mathrm{TM}(2 \mu \mathrm{g} / \mathrm{ml})-$

DHJSD $(\mu \mathrm{g} / \mathrm{ml}) 0$

PBA $(5 \mathrm{mM})$ -

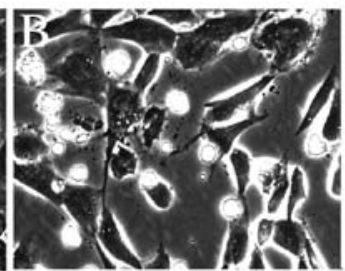

$+$

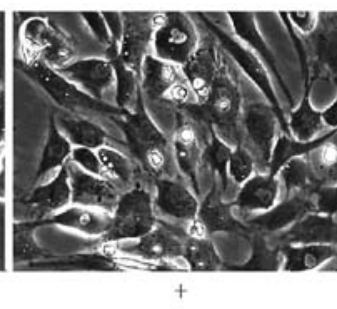

200

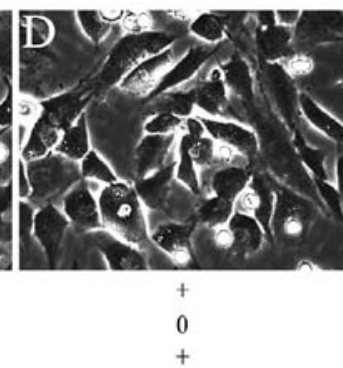

Figure 5. Duhuo Jisheng decoction (DHJSD) inhibits morphological changes in tunicamycin (TM)-exposed chondrocytes (magnification, x200). (A) Untreated cells. (B) TM-exposed chondrocytes. (C) TM-exposed chondrocytes treated by $200 \mu \mathrm{g} / \mathrm{ml}$ DHJSD for $24 \mathrm{~h}$. (D) TM-exposed chondrocytes treated by 4 -phenylbutyrate (PBA) for $24 \mathrm{~h}$, as the positive control.
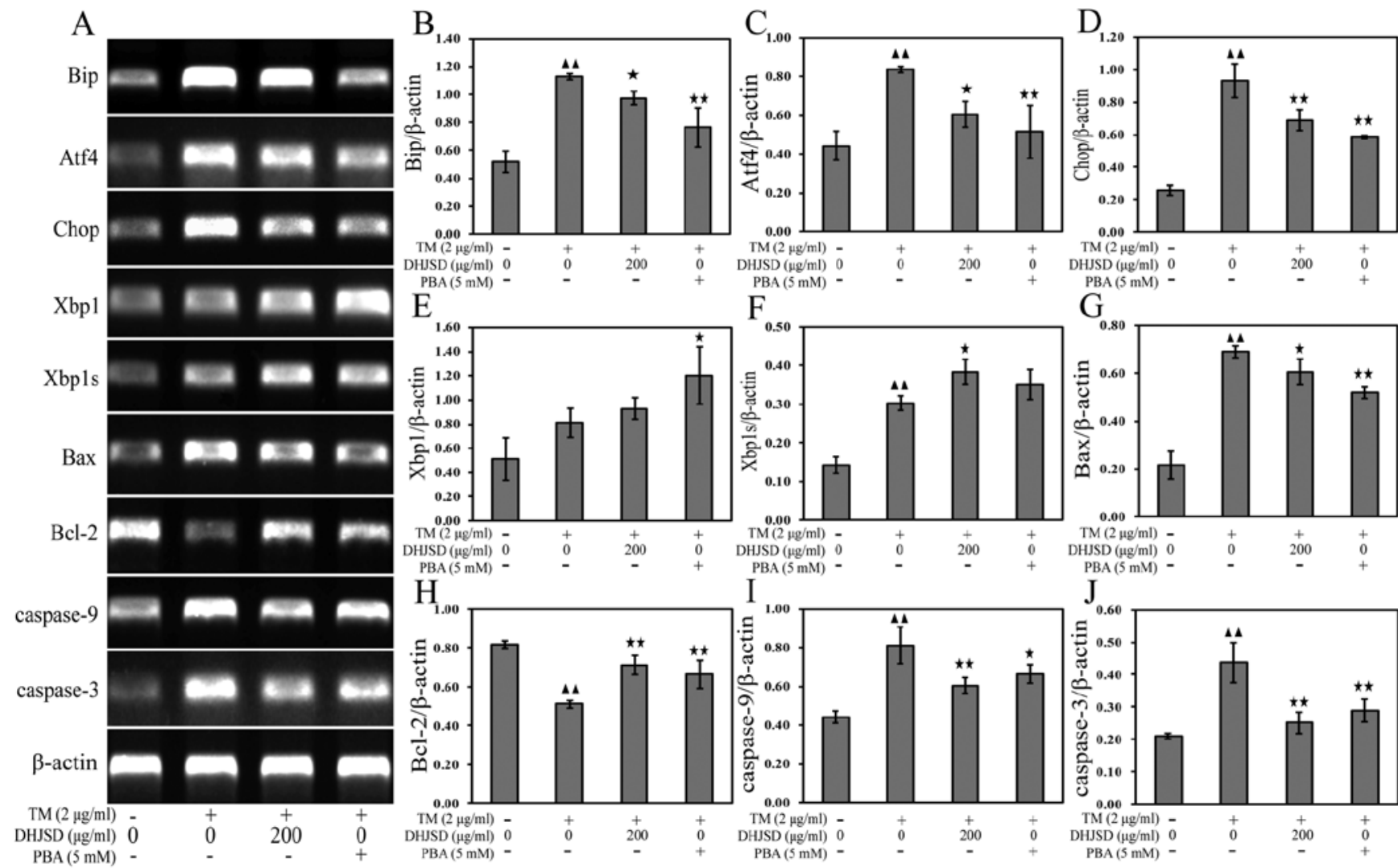

$\mathrm{F}$

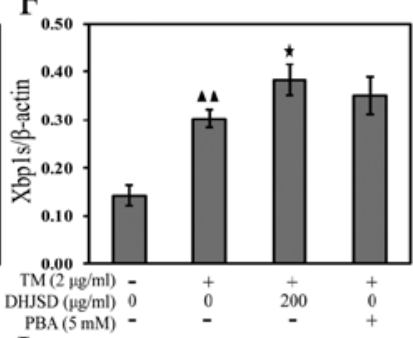

$\mathrm{G}$
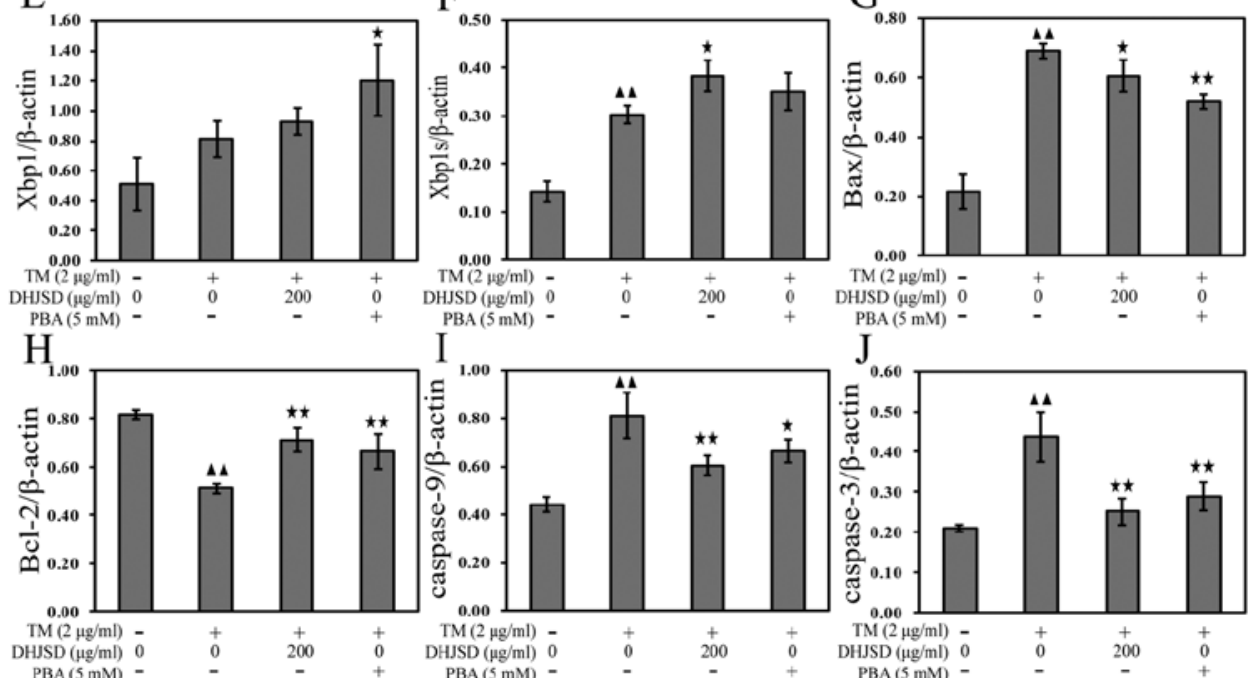

Figure 6. Duhuo Jisheng decoction (DHJSD) inhibits endoplasmic reticulum stress tunicamycin (TM)-induced chondrocyte apoptosis. Chondrocytes were treated with or without TM and DHJSD or 4-phenylbutyrate (PBA) in the presence of TM for $24 \mathrm{~h}$, and the associated mRNA was measured by (A) reverse transcription-polymerase chain reaction analysis. (B) mRNA expression of Bip, (C) Atf4, (D) Chop, (E) Xbp1, (F) Xbp1s, (G) Bax, (H) Bcl-2, (I) caspase-9 and (J) caspase-3. $\beta$-actin was used as the internal control for normalization. Values are means \pm standard deviation (SD) and SD is shown as vertical bars. ${ }^{\wedge} \mathrm{P}<0.01$ compared to untreated cells; ${ }^{* *} \mathrm{P}<0.01$ and ${ }^{*} \mathrm{P}<0.05$ compared to TM-induced chondrocytes.

DHJSD reduces the apoptosis of TM-exposed chondrocytes. To examine whether DHJSD enhanced TM-exposed chondrocyte viability by inhibiting apoptosis, the cells were determined by DAPI staining. The apoptotic cells exhibited typical changes, such as staining bright blue and condensed or fragmented nucleus. The typical changes were more observable in the TM-exposed chondrocytes than that of the TM-exposed chondrocytes treated with DHJSD or PBA (Fig. 3D-G).

DHJSD inhibits ER stress in induced by chondrocytes. To explore the role of DHJSD in chondrocytes of ER stress, the mRNA and protein expressions were examined by RT-PCR and western blot analysis, respectively. The results showed that the mRNA expression levels of Xbp1 and Xbp1s were increased, and the mRNA expression levels of Bip, Atf4 and Chop were decreased in TM-exposed chondrocytes treated with DHJSD or PBA compared to that in the TM-exposed chondrocytes $(\mathrm{P}<0.01$ and $\mathrm{P}<0.05)$ (Fig. 6A-F). The protein levels were similar to their respective mRNA expressions $(\mathrm{P}<0.01$ and $\mathrm{P}<0.05)$ (Fig. 7A-E), suggesting that DHJSD regulated ER stress in TM-exposed chondrocytes.

ER stress is known to induce the dysfunction of mitochondria, leading to caspase activation and subsequent apoptosis (22). A hallmark of apoptosis is the activation of caspases and the Bcl-2 family has a crucial role in regulating their engagement under ER stress (23). Bcl-2 antagonizes whereas Bax promotes ER stress-induced mitochondrial cytochrome $c$ release and caspase activation to induce apoptosis (24). To 
A

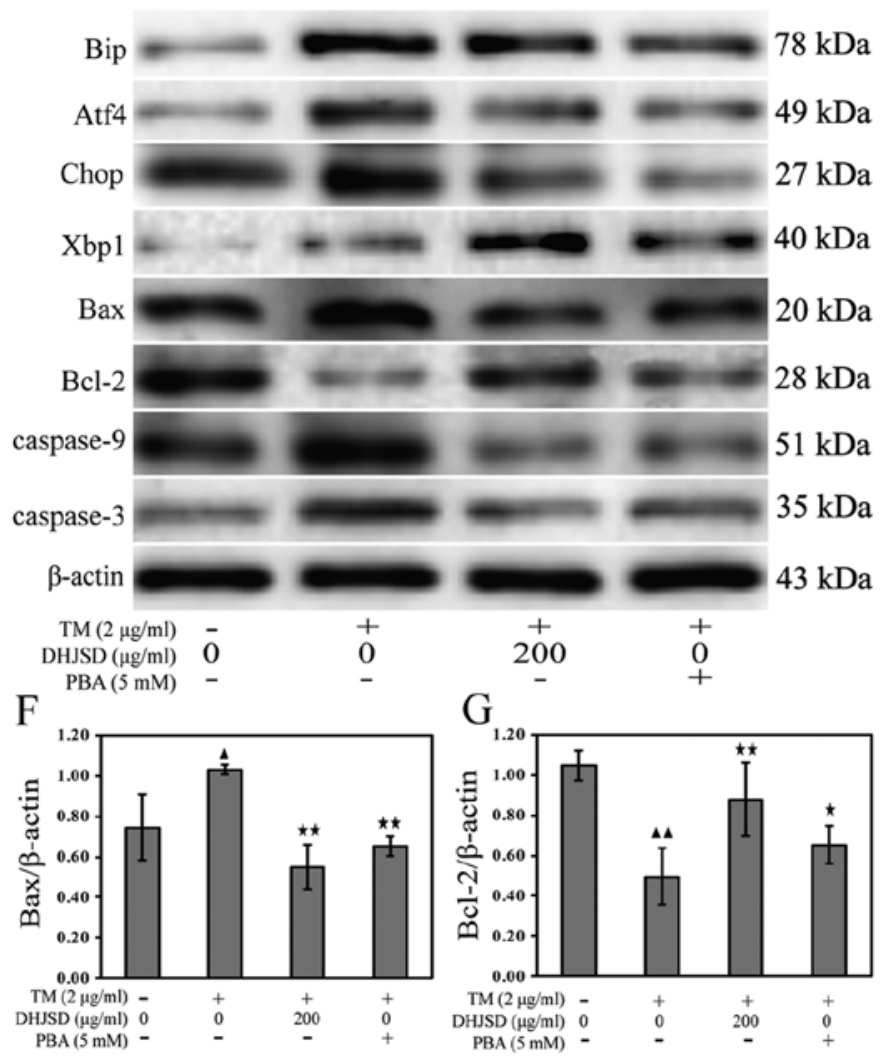

B

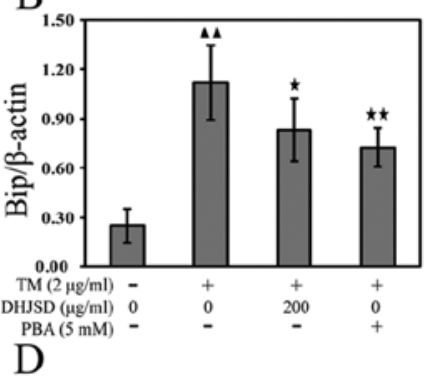

$\mathrm{D}_{1.20}$
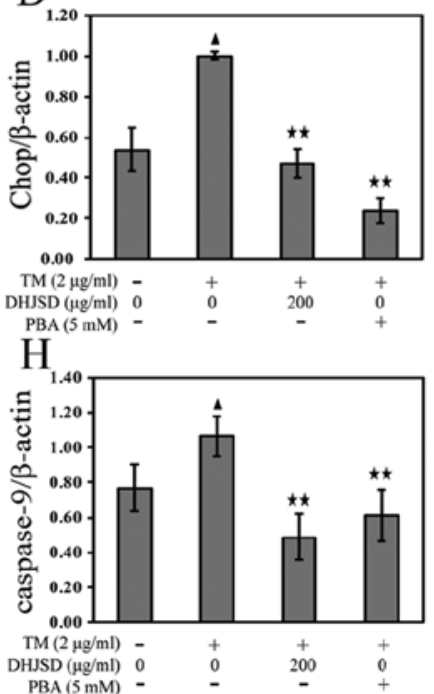

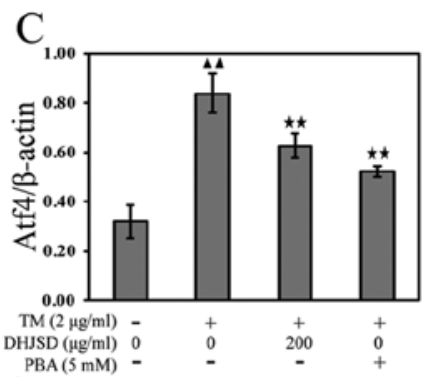

$\mathrm{EBA}$

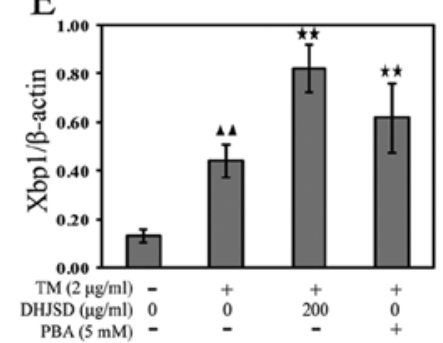

I

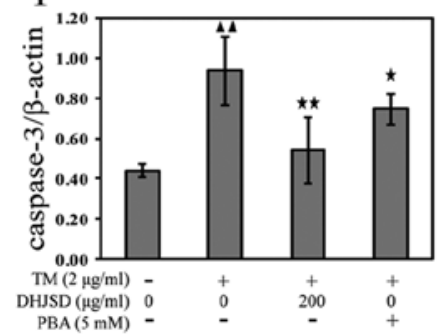

Figure 7. Duhuo Jisheng decoction (DHJSD) inhibits endoplasmic reticulum stress-induced apoptosis of tunicamycin (TM)-exposed chondrocytes. Chondrocytes were treated with or without TM and DHJSD or 4-phenylbutyrate (PBA) in the presence of TM for $24 \mathrm{~h}$, and the associated protein was measured by (A) western blot analysis. (B) Protein level of Bip, (C) Atf4, (D) Chop, (E) Xbp1, (F) Bax, (G) Bcl-2, (H) caspase-9 and (I) caspase-3. $\beta$-actin was used as the internal control for normalization. Values are means \pm standard deviation (SD) and SD is shown as vertical bars. ${ }^{\wedge} \mathrm{P}<0.01$ compared to untreated cells; ${ }^{* *} \mathrm{P}<0.01$ and ${ }^{*} \mathrm{P}<0.05$ compared to TM-exposed chondrocytes.

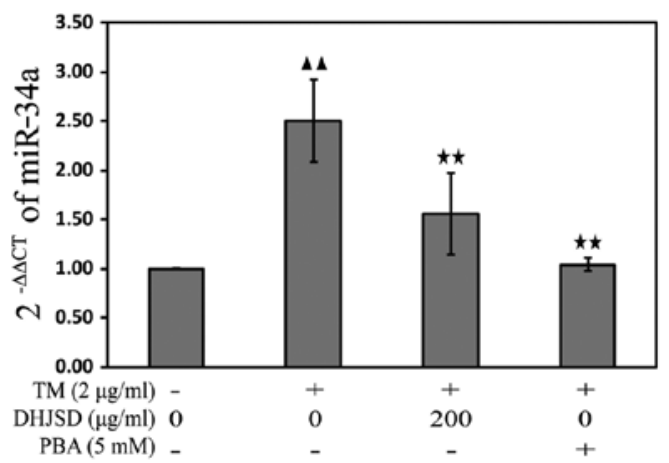

Figure 8. Duhuo Jisheng decoction (DHJSD) inhibits endoplasmic reticulum stress-induced apoptosis of tunicamycin (TM)-exposed chondrocytes by downregulating miR-34a. The miR-34a expression was analyzed by TaqMan microRNA assays, and U6 was the endogenous control. Fold changes were calculated by the formula $2^{-\Delta \Delta C t}$ relative to the expression in untreated cells. Values are means \pm standard deviation (SD) and SD is shown as vertical bars. ${ }^{\wedge} \mathrm{P}<0.01$ compared to untreated cells; ${ }^{* *} \mathrm{P}<0.01$ compared to TM-exposed chondrocytes.

gain insight into the mechanisms responsible for DHJSD on the apoptosis of TM-exposed chondrocytes, Bax, Bcl-2, caspase- 9 and caspase- $3 \mathrm{mRNA}$ and protein expressions were detected by RT-PCR and western blot analysis, respectively. The results showed that the expression of $\mathrm{Bcl}-2$ was increased, and the expression levels of Bax, caspase- 9 and caspase- 3 were decreased in TM-exposed chondrocytes treated with DHJSD or PBA compared to that in the TM-exposed chondrocytes $(\mathrm{P}<0.01$ and $\mathrm{P}<0.05)$ (Fig. 6A and G-J). The protein levels were similar to their respective mRNA expression levels $(\mathrm{P}<0.01$ and $\mathrm{P}<0.05$ ) (Fig. 7A and F-I), indicating that DHJSD inhibits apoptosis of TM-exposed chondrocytes by regulating ER stress.

DHJSD inhibits ER stress in the TM-exposed chondrocytes by downregulating miR-34a. miRNAs, a class of endogenous non-coding RNAs, regulate gene expression by binding to the 3'-untranslated region in their target mRNAs, resulting in either translational repression or degradation of target mRNA expression. To explore the mechanisms of DHJSD on the apoptosis of TM-exposed chondrocytes, the miR-34a expression was analyzed by the TaqMan microRNA assay. The results showed that the expression of miR-34a was downregulated in the TM-exposed chondrocytes treated with DHJSD or PBA compared with that in the TM-exposed chondrocytes $(\mathrm{P}<0.01)$ (Fig. 8), implying that DHJSD inhibits ER stress TM-exposed chondrocyte apoptosis by downregulating miR-34a.

\section{Discussion}

Natural products have been proved effective in treating OA by decreasing joint pain and dysfunction, and preventing and delaying the cartilage degeneration $(25,26)$. DHJSD contains a number of natural products with numerous chemical 
compounds that have been deemed to have multi-target agents and multi-components exerting their therapeutic function. However, the biological mechanisms of how DHJSD improves the clinical consequences of OA are not fully understood. The present results verified that DHJSD has multiple pathways to inhibit chondrocyte apoptosis.

To control the extract quality of DHJSD, UV was used to test the glucose and osthole concentrations, which belongs to polysaccharides and coumarins, respectively. Therefore, the extract quality of DHJSD was insured every time in the present study. The chondrocytes were identified by type II collagen immunohistochemical staining and toluidine blue staining, and the results indicated that the chondrocytes cultured in vitro can be established.

TM, an ER stress inducer, is an inhibitor of $N$-linked glycosylation and the formation of $N$-glycosidic protein-carbohydrate linkages (27). Therefore, TM-exposed chondrocytes were used as the ER stress apoptosis model. The effect of DHJSD on the viability of TM-exposed chondrocytes was examined by the MTT assay, and the results revealed that DHJSD increased cell viability. In addition, PBA, an ER stress inhibitor, aids in protein folding at the molecular level and prevents misfolded protein aggregation. Therefore, PBA was used as a positive control and the morphology changes of TM-exposed chondrocytes showed that TM-exposed chondrocyte survival could be enhanced by DHJSD or PBA. For further study, DAPI staining was used to explore whether DHJSD increased TM-exposed chondrocyte viability and enhanced TM-exposed chondrocyte survival by inhibiting apoptosis, and the results showed that DHJSD or PBA reduced TM-exposed chondrocyte apoptosis. It remains to be determined whether DHJSD reduced TM-exposed chondrocyte apoptosis by regulating ER stress.

The ER is a sophisticated lumen where protein synthesis, folding and maturation occur. Perturbation of these processes in the pathological states results in ER stress, and activate a complex signaling network (28). Bip, an ER chaperone protein, alleviates ER stress and maintains ER function by binding to the incompletely folded proteins or are unfolded to prevent the interaction of these proteins with surrounding molecules, and whose expression induces ER stress (29). During ER stress, Bip away from the UPR sensors Perk (the first responses of the cell to ER stress), Atf6 and Ire1, results in the three sensors phosphorylation (27). Perk is responsible for phosphorylating the translation initiation factor, eIF $2 \alpha$, that enhances the translation of Atf4 (30). Atf4 induces the pro-apoptotic events by activating Chop, a key factor of ER stress, whose overexpression evokes cell apoptosis $(31,32)$, and all the three sensors phosphorylation can induce transcription of Chop in response to ER stress. By contrast, upon activation of the UPR, Irel autophosphorylation activates and serves as endoribonuclease, which removes 26 ribonucleotides from the Xbp1 mRNA that undergoes transcription by Atf6 activation, allowing production of the Xbp1 protein into Xbp1 spliced form (Xbpls) mRNA to generate a more potent transcription factor, Xbpls, a key transcriptional regulator that restores ER function by refolding or reducing misfolded proteins that have accumulated in ER lumen (33). The present results showed that DHJSD or PBA regulated ER stress by decreasing Bip, Atf4 and Chop, and helped to restore ER function by increasing Xbpl and Xbpls.
Mitochondria are recognized as the central regulator of apoptotic cell death, and ER-mitochondrial cross talk may mediate stress signals between these compartments (34). Accumulating evidence indicates that ER stress results in apoptosis by regulating the $\mathrm{Bcl}-2$ family proteins that regulate the release of calcium from the ER and the release of pro-apoptotic factors from mitochondria (35-37). Bax, one of the Bcl-2 family proteins is recruited to the ER surface and the mitochondria to induce apoptosis, whereas the anti-apoptotic Bcl-2 can prevent ER stress-mediated apoptosis (36). Bax and pro-apoptotic Bcl-2 are switched on by the Irel pathway, and Chop can repress the pro-survival gene Bcl-2 (38-40). Caspases, a family of cysteine proteases, act as common death effector molecules in various forms of apoptosis and are involved in initiating and completing the final execution of the cell (32). Caspase-12 is important in the context of ER stress-mediated apoptosis and its activation cleaves pro-caspase-9, which in turn activates caspase-3, thus leading to cell death (41). The present results demonstrated that DHJSD or PBA inhibited ER stress apoptosis by increasing Bcl-2, whereas decreasing Bax, caspase-9 and caspase-3.

miRNAs regulate protein expression by degrading target mRNA or inhibiting translation, resulting in complementary matching between miRNAs and specific sites in target mRNAs (42), correlate with human disease and have a potential as therapeutic targets (43-45). Emerging evidence suggests miRNAs control the balance of pro-survival and pro-apoptotic signals by acting at different steps in each arm of the pathway to regulate ER stress (46). To identify the possible mechanisms, the expression of miR-34a was examined by RT-qPCR and results showed that miR-34a was markedly downregulated in TM-exposed chondrocytes treated with DHJSD or PBA.

In conclusion, the present results expand the significant roles of DHJSD in reducing chondrocyte apoptosis by inhibiting the ER stress apoptotic pathway, suggesting it may be a potential drug for the treatment of OA. However, due to the limitations in vitro and the exact mechanism implicated in the regulation of ER stress in chondrocyte by DHJSD remains unclear. Further studies are required to confirm the effects in vivo, and a small interfering RNA inhibitor could be used to confirm the precise mechanisms.

\section{Acknowledgements}

The present study was supported by the National Natural Science Foundation of China (grant no. 81373818), the Special Research Fund for Doctor Discipline in College (20123519110001), the Young and Middle-aged Core Personnel Training Project of Fujian Province Health Department (grant no. 2014-ZQN-JC-29), and the Young-aged Teacher Educational and Scientific Research Project of Fujian Province (grant no. JA12165), the Developmental Fund of Chen Keji Integrative Medicine (grant nos. CKJ2014014, CKJ2014001 and CKJ2015009), the Key Project of Fujian Provincial Department of Science and Technology (grant no. 2014Y0064) and the Natural Science Foundation of Fujian Province (grant no. 2014J01357).

\section{References}

1. Bijlsma JW, Berenbaum F and Lafeber FP: Osteoarthritis: An update with relevance for clinical practice. Lancet 377 : 2115-2126, 2011. 
2. Loeser RF: Aging and osteoarthritis: The role of chondrocyte senescence and aging changes in the cartilage matrix. Osteoarthritis Cartilage 17: 971-979, 2009.

3. Mistry D, Oue Y, Chambers MG, Kayser MV and Mason RM: Chondrocyte death during murine osteoarthritis. Osteoarthritis Cartilage 12: 131-141, 2004.

4. Thomas CM, Fuller CJ, Whittles CE and Sharif M: Chondrocyte death by apoptosis is associated with cartilage matrix degradation. Osteoarthritis Cartilage 15: 27-34, 2007.

5. Gorman AM, Healy SJ,Jäger R and Samali A: Stress management at the ER: Regulators of ER stress-induced apoptosis. Pharmacol Ther 134: 306-316, 2012.

6. Takada K, Hirose J, Senba K, Yamabe S, Oike Y, Gotoh T and Mizuta H: Enhanced apoptotic and reduced protective response in chondrocytes following endoplasmic reticulum stress in osteoarthritic cartilage. Int J Exp Pathol 92: 232-242, 2011.

7. Bernales S, Papa FR and Walter P: Intracellular signaling by the unfolded protein response. Annu Rev Cell Dev Biol 22: 487-508, 2006.

8. Walter P and Ron D: The unfolded protein response: From stress pathway to homeostatic regulation. Science 334: 1081-1086, 2011

9. Kim R, Emi M, Tanabe K and Murakami S: Role of the unfolded protein response in cell death. Apoptosis 11: 5-13, 2006.

10. Yang L, Carlson SG, McBurney D and Horton WE Jr: Multiple signals induce endoplasmic reticulum stress in both primary and immortalized chondrocytes resulting in loss of differentiation, impaired cell growth, and apoptosis. J Biol Chem 280: 31156-31165, 2005.

11. Yang L, McBurney D, Tang SC, Carlson SG and Horton WE Jr: A novel role for Bcl-2 associated-athanogene-1 (Bag-1) in regulation of the endoplasmic reticulum stress response in mammalian chondrocytes. J Cell Biochem 102: 786-800, 2007.

12. Lafferty-Whyte K, Cairney CJ, Jamieson NB, Oien KA and Keith WN: Pathway analysis of senescence-associated miRNA targets reveals common processes to different senescence induction mechanisms. Biochim Biophys Acta 1792: 341-352, 2009.

13. Kloosterman WP and Plasterk RH: The diverse functions of microRNAs in animal development and disease. Dev Cell 11: 441-450, 2006

14. Abouheif MM, Nakasa $T$, Shibuya $H$, Niimoto $T$, Kongcharoensombat W and Ochi M: Silencing microRNA-34a inhibits chondrocyte apoptosis in a rat osteoarthritis model in vitro. Rheumatology (Oxford) 49: 2054-2060, 2010.

15. Zheng CS, Xu XJ, Ye HZ, Wu GW, Li XH, Huang SP and Liu XX: Computational approaches for exploring the potential synergy and polypharmacology of Duhuo Jisheng decoction in the therapy of osteoarthritis. Mol Med Rep 7: 1812-1818, 2013.

16. Liu F, Liu G, Liang W, Ye H, Weng X, Lin P, Li H, Chen J, Liu X and Li X: Duhuo Jisheng decoction treatment inhibits the sodium nitroprussiate induced apoptosis of chondrocytes through the mitochondrial dependent signaling pathway. Int J Mol Med 34: $1573-1580,2014$

17. Li H, Li X, Liu G, Chen J, Weng X, Liu F, Xu H, Liu X and Ye H: Bauhinia championi (Benth.) Benth. polysaccharides upregulate Wnt $/ \beta$-catenin signaling in chondrocytes. Int J Mol Med 32: 1329-1336, 2013

18. Hu G, Huang K, Yu J, Gopalakrishna-Pillai S, Kong J, Xu H, Liu Z, Zhang K, Xu J, Luo Y, et al: Identification of miRNA signatures during the differentiation of hESCs into retinal pigment epithelial cells. PLoS One 7: e37224, 2012.

19. Yu F, Li X, Cai L, Li H, Chen J, Wong X, Xu H, Zheng C, Liu X and Ye H: Achyranthes bidentata polysaccharides induce chondrocyte proliferation via the promotion of the G1/S cell cycle transition. Mol Med Rep 7: 935-940, 2013.

20. Li X, Peng J, Wu M, Ye H, Zheng C, Wu G, Xu H, Chen X and Liu X: BMP2 promotes chondrocyte proliferation via the Wnt//-catenin signaling pathway. Mol Med Rep 4: 621-626, 2011

21. Li X, Du M, Liu X, Chen W, Wu M, Lin J and Wu G: Millimete wave treatment promotes chondrocyte proliferation by upregulating the expression of cyclin-dependent kinase 2 and cyclin $\mathrm{A}$ Int J Mol Med 26: 77-84, 2010

22. Zong WX, Li C, Hatzivassiliou G, Lindsten T, Yu QC, Yuan J and Thompson CB: Bax and Bak can localize to the endoplasmic reticulum to initiate apoptosis. J Cell Biol 162: 59-69, 2003

23. Riedl SJ and Shi Y: Molecular mechanisms of caspase regulation during apoptosis. Nat Rev Mol Cell Biol 5: 897-907, 2004.

24. Gupta S, Cuffe L, Szegezdi E, Logue SE, Neary C, Healy S and Samali A: Mechanisms of ER Stress-Mediated Mitochondria Membrane Permeabilization. Int J Cell Biol 2010: 170215, 2010.
25. Hua B, Abbas E, Hayes A, Ryan P, Nelson L and O'Brien K: Reliability of Chinese medicine diagnostic variables in the examination of patients with osteoarthritis of the knee. J Altern Complement Med 18: 1028-1037, 2012.

26. Wang X, Wei S, Liu T, Pang J, Gao N, Ding D, Duan T, Cao Y, Zheng $\mathrm{Y}$ and Zhan $\mathrm{H}$ : Effectiveness, medication patterns, and adverse events of traditional chinese herbal patches for osteoarthritis: A systematic review. Evid Based Complement Alternat Med 2014: 343176, 2014.

27. Lin JH, Li H, Yasumura D, Cohen HR, Zhang C, Panning B, Shokat KM, Lavail MM and Walter P: IRE1 signaling affects cell fate during the unfolded protein response. Science 318: 944-949, 2007.

28. Ron D and Walter P: Signal integration in the endoplasmic reticulum unfolded protein response. Nat Rev Mol Cell Biol 8: 519-529, 2007.

29. Yeung BH, Kwan BW, He QY, Lee AS, Liu J and Wong AS: Glucose-regulated protein 78 as a novel effector of BRCA1 for inhibiting stress-induced apoptosis. Oncogene 27: 6782-6789, 2008.

30. DuRose JB, Scheuner D, Kaufman RJ, Rothblum LI and Niwa M: Phosphorylation of eukaryotic translation initiation factor $2 \alpha$ coordinates rRNA transcription and translation inhibition during endoplasmic reticulum stress. Mol Cell Biol 29: 4295-4307, 2009.

31. Hotamisligil GS: Endoplasmic reticulum stress and the inflammatory basis of metabolic disease. Cell 140: 900-917, 2010.

32. Sovolyova N, Healy S, Samali A and Logue SE: Stressed to death - mechanisms of ER stress-induced cell death. Biol Chem 395: 1-13, 2014

33. Yoshida H, Matsui T, Yamamoto A, Okada T and Mori K: XBP1 mRNA is induced by ATF6 and spliced by IRE1 in response to ER stress to produce a highly active transcription factor. Cell 107: 881-891, 2001.

34. Ouyang YB, Xu LJ, Emery JF, Lee AS and Giffard RG: Overexpressing GRP78 influences $\mathrm{Ca}^{2+}$ handling and function of mitochondria in astrocytes after ischemia-like stress. Mitochondrion 11: 279-286, 2011.

35. Chipuk JE, Moldoveanu T, Llambi F, Parsons MJ and Green DR: The BCL-2 family reunion. Mol Cell 37: 299-310, 2010.

36. Scorrano L, Oakes SA, Opferman JT, Cheng EH, Sorcinelli MD, Pozzan T and Korsmeyer SJ: BAX and BAK regulation of endoplasmic reticulum $\mathrm{Ca}^{2+}$ : A control point for apoptosis. Science 300: 135-139, 2003

37. Rodriguez DA, Zamorano S, Lisbona F, Rojas-Rivera D, Urra H, Cubillos-Ruiz JR, Armisen R, Henriquez DR, Cheng EH, Letek $\mathrm{M}$, et al: $\mathrm{BH} 3$-only proteins are part of a regulatory network that control the sustained signalling of the unfolded protein response sensor IRE1 $\alpha$. EMBO J 31: 2322-2335, 2012.

38. Hetz C, Bernasconi P, Fisher J, Lee AH, Bassik MC, Antonsson B, Brandt GS, Iwakoshi NN, Schinzel A, Glimcher LH, et al: Proapoptotic BAX and BAK modulate the unfolded protein response by a direct interaction with IRE1 $\alpha$. Science 312: 572-576, 2006

39. Rodriguez D, Rojas-Rivera D and Hetz C: Integrating stress signals at the endoplasmic reticulum: The BCL-2 protein family rheostat. Biochim Biophys Acta 1813: 564-574, 2011.

40. Chen Y and Brandizzi F: IRE1: ER stress sensor and cell fate executor. Trends Cell Biol 23: 547-555, 2013.

41. Morishima N, Nakanishi K, Takenouchi H, Shibata $T$ and Yasuhiko Y: An endoplasmic reticulum stress-specific caspase cascade in apoptosis. Cytochrome c-independent activation of caspase-9 by caspase-12. J Biol Chem 277: 34287-34294, 2002.

42. Friedman RC, Farh KK, Burge CB and Bartel DP: Most mammalian mRNAs are conserved targets of microRNAs. Genome Res 19: 92-105, 2009.

43. Tili E, Michaille JJ, Costinean S and Croce CM: MicroRNAs, the immune system and rheumatic disease. Nat Clin Pract Rheumatol 4: 534-541, 2008 .

44. O'Connell RM, Rao DS, Chaudhuri AA and Baltimore D: Physiological and pathological roles for microRNAs in the immune system. Nat Rev Immunol 10: 111-122, 2010.

45. Dai R and Ahmed SA: MicroRNA, a new paradigm for understanding immunoregulation, inflammation, and autoimmune diseases. Transl Res 157: 163-179, 2011.

46. Bartoszewska S, Kochan K, Madanecki P, Piotrowski A, Ochocka R, Collawn JF and Bartoszewski R: Regulation of the unfolded protein response by microRNAs. Cell Mol Biol Lett 18: 555-578, 2013. 